\title{
Diferencias en la realización e interpretación de la espirometría del preescolar y en el niño mayor
}

\author{
Dra. Marcela Linares' \\ 1 Pediatra Especialista en Enfermedades Respiratorias. Clinica Indisa. Univesidad Finis Terrae.
}

\section{DIFFERENCES ON SPIROMETRY STANDARDIZATION BETWEEN PRES- CHOOL AND SCHOOL CHILDREN}

Spirometry must meet international standardizations criteria to be valid. Those criterias are classically described to be applied on starting from 6 years old children. In recent years, there have been numerous publications demonstrating that spirometry can be performed in preschool children, whenever the standardization is adapted to this age group. In this revision we describe the differences to perform and interpret spirometry between preschool children and children over 6 years old.

Key words: Spirometry; preschool children; reference standards.

\section{RESUMEN}

Para que la espirometría sea válida, debe cumplir con los criterios establecidos en estandarizaciones internacionales, las que clásicamente están descriptas para ser aplicadas a partir de los 6 años. En los últimos años, han surgido numerosas publicaciones que demuestran que la espirometría puede ser realizada en preescolares, siempre que se adapten las exigencias a este grupo etario. Se describen las diferencias entre el niño preescolar y el niño mayor de 6 años, para realizar e interpretar la espirometría.

Palabras clave: Espirometría, preescolar, estándares de referencia.

\section{INTRODUCCIÓN}

La espirometría tiene un valor indiscutible en la evaluación de la función pulmonar debido a su masiva utilización en forma estandarizada en distintas edades, disponibilidad de valores de referencia y facilidad y familiaridad en la interpretación de los resultados. Permite obtener un seguimiento longitudinal con la misma técnica de función pulmonar en las patologías que comienzan en edades tempranas de la vida(I).

La correcta interpretación de la espirometría depende del acucioso seguimiento de los criterios de aceptabilidad y repetibilidad estandarizados por la Sociedad Americana de Tórax (ATS) y por la Sociedad Respiratoria Europea (ERS), lo que permite minimizar la variabilidad interindividial e intraindividual de la prueba ${ }^{(2)}$. Esta estandarización está definida para ser aplicada desde los 6 años.

Las consideraciones del equipo, ambiente y operador son especiales para el grupo en edad pre-escolar. El espirómetro debe tener la capacidad de evaluar flujos instantáneos con una adecuación de más menos $5 \%$ y poseer el menor espacio muerto posible ${ }^{(I)}$. El software debe disponer de incentivos

Correspondencia:

Dra. Marcela Linares P.

E-mail:m.linares@manquehue.net

Conflicto de interés: Autor declara no poseer conflicto de interés. animados que permitan una exhalación rápida y prolongada y la posibilidad de que las curvas flujo/volumen y volumen/ tiempo sean analizadas en el momento en que se realiza la maniobra $O$ antes de comenzar la siguiente. Esto permite evaluar la calidad del examen e incentivar al niño a corregir los errores de la curva anterior ${ }^{(l)}$. El operador debe saber ganarse la confianza del preescolar y ser capaz de obtener las curvas sin estresar al niño. Con el mismo objetivo, el ambiente debe ser adecuado para niños, contar juguetes y videos infantiles. Cuando el preescolar no ha realizado espirometrías previamente, puede ser útil el empleo de un tiempo de entrenamiento, durante el cual el niño juegue con la pieza bucal, antes de comenzar a registrar las curvas realizadas.

El niño pequeño tiene una capacidad de atención de pocos minutos, se distrae con facilidad y no es capaz de coordinar varias instrucciones al mismo tiempo, por lo que durante la espiración forzada no puede espirar rápido, fuerte y sostener ese esfuerzo por un período de tiempo, en general sólo cumple con una de estas condiciones ${ }^{(3)}$. Por otro lado, el volumen de la capacidad vital de los preescolares es expulsado en su totalidad en alrededor de I segundo((1,3,4). Estas características fisiológicas explican por qué la estandarización de la espirometría diseñada para adultos, no puede ser aplicada en preescolares, e incluso sea difícil de cumplir para los niños mayores, aún con experiencia previa de haber realizado una espirometría ${ }^{(3,5)}$.

Han surgido varias publicaciones que demuestran que la espirometría puede ser realizada en forma exitosa por preescolares, siempre y cuando las condiciones de estanda- 
rización y aceptabilidad se adapten según las capacidades de estos pequeños ${ }^{(3,5-15)}$. El rendimiento de la espirometría en niños preescolares varía entre un 60 a 90\% según distintos trabajos y es directamente proporcional a la edad ${ }^{(5-15)}$. A pesar de la falta de colaboración que habitualmente se encuentra en estos pequeños, el promedio del coeficiente de variación de Volumen Espirado al Primer Segundo $\left(V_{E F}\right)$ y Capacidad Vital Forzada (CVF) es de 3\%, de Volumen Espirado a 0,5 segundos $\left(\mathrm{VEF}_{0.5}\right)$ es de $4 \%$ y I I\% para Flujo Espiratorio Forzado entre el 25 y $75 \%$ de la CVF $\left(\mathrm{FEF}_{25-75}\right)$ valores semejantes a lo encontrado en adultos ${ }^{(7)}$.

A continuación se detallan las diferencias para realizar e interpretar la espirometría entre preescolares y niños mayores.

\section{REALIZACIÓN DEL EXAMEN}

Para la realización con éxito de la espirometría en preescolares es conveniente limitar el tiempo de permanencia en el laboratorio durante el cuál el niño realice todas las maniobras posibles, no exigir el uso de clip nasal, evaluar individualmente la utilización de programas de incentivo, y grabar todas las maniobras posibles ${ }^{(1,5)}$.

A pesar de que las estandarizaciones enfatizan el uso de clip nasal en niños mayores ${ }^{(2)}$, no es necesario exigir su uso en preescolares, por que es un elemento distractor y a veces asusta al niño, y se ha observado que no hay diferencias en los valores de VEF, y CVF obtenidos con o sin pinza nasa( ${ }^{(16)}$.

El uso de programas animados por computadora para incentivar al niño a realizar maniobras aceptables y repetibles ha sido evaluado en algunas publicaciones con resultados dispares $^{(9,17)}$. El programa que consiste en apagar velas permite al niño lograr mejores Flujos Espiratorios Máximos (FEM), pero no se facilita una espiración sostenida en el tiempo, por lo que los valores de CVF obtenidos con este programa son menores. No se recomienda el uso de los programas de incentivo de rutina, ya que su efectividad es variable para cada niño y para cada tipo de software utilizado(16).

La ATS y ERS recomiendan realizar en el adulto y niños mayores un máximo de 8 maniobras ${ }^{(2)}$. El preescolar es capaz de realizar un número mayor de maniobras en menos tiempo, ya que su tiempo espiratorio es menor. Esto permite aumentar la posibilidad de obtener una espirometría válida, ya que a mayor número de maniobras hay mayor efecto de entrenamiento y por lo tanto mayor posibilidad de que alguna de ellas sea adecuada para su interpretación ${ }^{(5)}$. Debido a que el preescolar tiene una capacidad de atención limitada, se aconseja limitar el tiempo de permanencia en el laboratorio a I 5 min como máximo ${ }^{(4)}$. Idealmente se deben grabar todas las maniobras realizadas por el niño, para hacer una selección en un tiempo posterior, aunque lamentablemente esto no puede lograrse con todos los equipos ${ }^{(5)}$. En ese caso se pueden grabar al menos las 3 curvas visiblemente mejores, las que se definen como un FEM claramente determinado y de rápido ascenso, espiración sin artefactos ni evidencia de inspiración temprana o cese brusco de la espiración y se deben excluir las curvas que denotan un pobre esfuerzo, cierre glótico, tos y obstrucción de la pieza bucal (Figura I)(1).

A diferencia que el niño mayor y el adulto, para el preescolar es más fácil realizar la maniobra de pie.

\section{CRITERIO DE ACEPTABILIDAD DEL INICIO DE LA CURVA}

Igual que en el niño mayor, se debe considerar que la curva tenga un FEM visualmente evidente y de ascenso rápido, que la parte más alta de la curva sea triangular y no

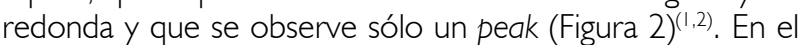
preescolar, no se aconseja utilizar el tiempo de ascenso del FEM menor a 0, I segundo descrito por Miller como criterio de aceptabilidad, ya que se ha demostrado que ninguno de ellos es capaz de lograrlo(15).

El Volumen de extrapolación retrógrada (VER) aceptable para preescolares está entre el 10 y 12\% u $80 \mathrm{ml}$ de la CVF y no de $5 \%$ como en mayores ${ }^{(1,5,15)}$.

\section{CRITERIO DE ACEPTABILIDAD DEL FINAL DE LA CURVA}

La ATS define que la espiración debe continuar hasta que se observe una meseta de al menos I segundo en la curva volumen tiempo y/o un tiempo espiratorio de al menos 3 segundos en el niño menor de 10 años y mayor de 6 segundos en el niño mayor de 10 años ${ }^{(2)}$. El tiempo espiratorio normal del preescolar es de alrededor de I segundo y es directamente proporcional a la edad, de tal forma que el 30 y $70 \%$ de los niños menores y mayores de 5 años respectivamente pueden mantener la espiración durante al menos I segundo $(1,5,7,11,15)$. Se ha sugerido que en este grupo etario, el tiempo espiratorio no sea un criterio de no aceptación de la prueba y sólo se debe reportar en el informe ${ }^{(1)}$. El preescolar puede vaciar sus pulmones completamente en un segundo debido a que tienen un volumen pulmonar pequeño(4,5). Esto implica que el VEF, y la CVF en algunas ocasiones pueden tener el mismo valor, por lo que el VEF, y la relación $\mathrm{VEF}_{1} /$ CVF no servirían para evaluar obstrucción bronquial, siendo los flujos espirados al 0,5 y 0,75 de la CVF y la relación $\operatorname{VEF}_{0,5} / C V F$ más útiles para evaluar a estos niños ${ }^{(5,14,15,18)}$.

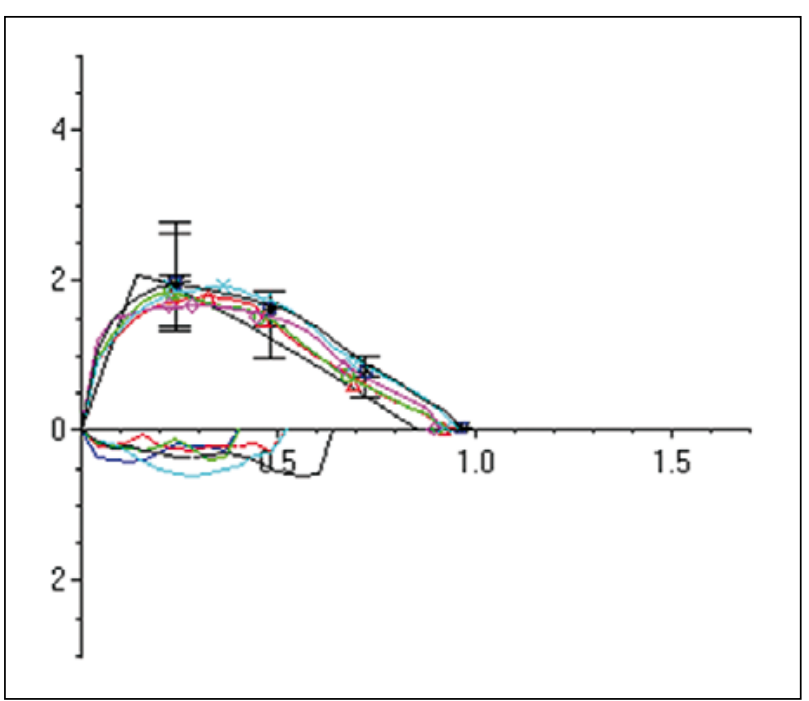

Figura I. Curvas grabadas, visiblemente reproducibles. 
Hay que tener presente que muchos niños no logran espirar ni siquiera durante un segundo, por lo que no podemos contar con $\mathrm{VEF}$, como parámetro de evaluación. Algunos equipos arrojan un valor extrapolado de $\mathrm{VEF}$, cuando hay un tiempo espiratorio menor de I segundo y en algunos casos sobreestiman el tiempo espiratorio, cuando el niño quita su boca de la pieza bucal antes de inspirar nuevamente o que el operador detenga el registro(15).

La finalización de la prueba en forma de meseta no puede exigirse en preescolares, sobre todo en los más pequeños, que tienden a finalizar bruscamente la espiración, aún cuando han logrado una exhalación completa (Figura 2) $)^{(1,5)}$. Se puede elegir como punto de corte que no finalicen bruscamente en flujos > al $10 \%$ del FEM o en flujos menores a $200 \mathrm{ml} /$ seg (Figura 2) $)^{(1,5,6,7,9)}$. El inconveniente de este criterio es que el valor no se encuentra disponible en el software en forma automática y hay que hacer un cálculo manual.

\section{REPETIBILIDAD}

Los preescolares pueden lograr un 10\% de repetibilidad pero no un $5 \%$ como lo exige ATS para adultos $(1,5,8,15)$. La diferencia de $150 \mathrm{ml}$ entre las dos mejores mediciones de CVF y VEF, que se aplica en adultos como criterio de repetibilidad, no es recomendable en preescolares, ya que son volúmenes muy altos para el volumen pulmonar total de estos pequeños ${ }^{(1,2)}$.

Se ha comprobado al aplicar una presión negativa a la vía aérea abierta durante le espiración forzada en niños de 3 a 6 años, se obtienen curvas que no cambian significativamente unas de otras, sólo se observa una mejoría del VEF, del 2\%, lo que es clínicamente despreciable, lo que significa que la limitación al flujo puede ser alcanzada a esta edad ${ }^{(10)}$. Por lo tanto, una sola curva que ha alcanzado la limitación al flujo puede ser adecuada para definir la función pulmonar. Es por este motivo que el hecho de no alcanzar criterio de repetibilidad no invalidaría el examen, de la misma forma como ocurre en el adulto(1,5).

\section{INTERPRETACIÓN}

Una vez obtenidas curvas aceptables y repetibles, la interpretación de los resultados de preescolares no difiere de los niños mayores.

No se dispone de valores de referencia nacionales para preescolares. De los valores de referencia internacionalmente, los de Eigen y cols y Zapletal y cols, no tienen las variables $\operatorname{VEF}_{0.5}$ ó 0,75 que se necesita para su interpretación (7,12). EI grupo de aproximadamente 600 niños evaluados por Nystad y cols, incluyeron algunos niños asmáticos ${ }^{(8)}$. Pérez Yarza y cols publicaron valores e referencia del grupo CANDELA, los que tienen el inconveniente de no tener los resultados necesarios para generar el valor del P5 y ser niños sin ningún tipo de exposición a contaminantes inhalados, lo que difiere de la realidad de varias ciudades de Chile ${ }^{(14)}$. Recientemente se han generado valores de referencia multiétnicos que abarcan desde los 3 a los 90 años. Aunque no se incluyeron preescolares

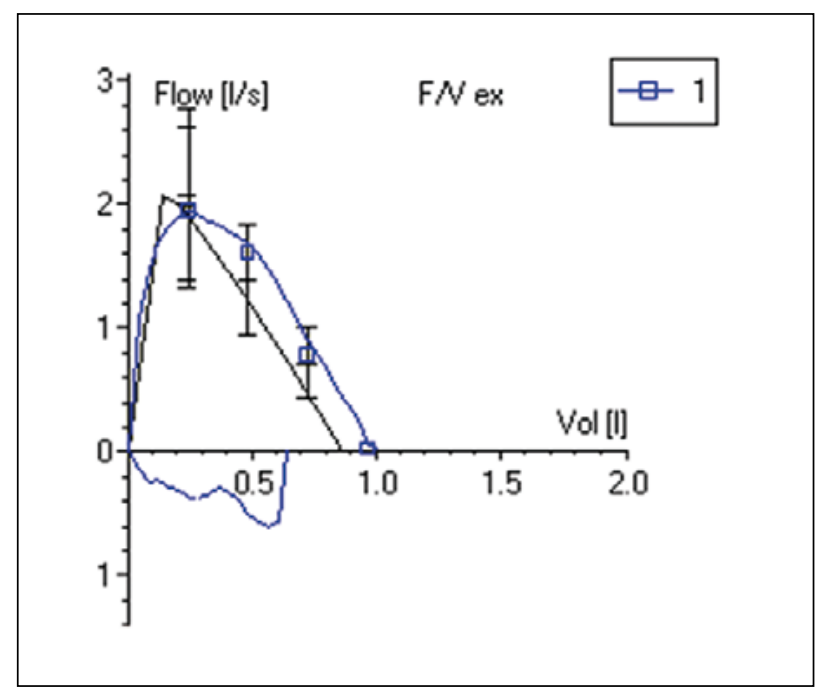

Figura 2. Curva flujo-volumen de preescolar. Características normales: Ascenso rápido, forma triangular, se observa sólo un peak, sin artefactos y cese de flujo menor a $200 \mathrm{ml} / \mathrm{seg}$.

chilenos, tienen la ventaja de ser valores lineales a lo largo de todos los rangos etarios y tienen el valor de $\mathrm{VEF}_{0.75}$, el P5 y Z score disponibles para su interpretación ${ }^{(19)}$. De todas formas, para decidir qué valor de referencia utilizar en la interpretación de la espirometría de preescolares, cuando no se dispone de valores propios, se puede correlacionar 30 niños sanos con los valores de referencia disponibles internacionalmente y seleccionar los que mejor representan a esta población ${ }^{(19)}$.

Independientemente de cuál sea el valor de referencia seleccionado se debe disponer del límite inferior o P5 (Percentil 5) y Z score de cada variable para su correcta interpretación. Se considera normal a los valores que se encuentran dentro de más menos I,64 desvíos estándar de la media (Z score) o por encima del $\mathrm{P} 5^{(1)}$.

Por el momento, en la espirometría del preescolar es muy útil la interpretación de la respuesta broncodilatadora, la que se puede considerar significativa como $12 \%$ en $\mathrm{VEF}_{0,5}$ y $30 \%$ en $\mathrm{FEF}_{25-75}$, siempre y cuando el coeficiente de repetibilidad esté por debajo de estos valores ${ }^{(20)}$.

\section{COMENTARIO FINAL}

La espirometría es un examen de alto rendimiento en preescolares cuando se adecuan las exigencias de estandarización. Para aumentar la utilidad de la espirometría en preescolares es necesario contar con valores de referencia nacionales, de los cuales aún no disponemos.

\section{REFERENCIAS}

I. An Official American Thoracic Society/European Respiratory Society Statement: Pulmonary Function Testing in Preeschool Children. Am J Respir Crit Care Med 2007; I75: I 304-45.

2. Miller M, Hankinson J, Brusasco V, Burgos F, Casaburi R, Coates A, et al. SERIES "ATS/ERS task force: standardisation of lung function 
testing: Standardisation of spirometry". Eur Respir J 2005; 26: 31 938.

3. Kanengiser S, Dozor AJ. Forced expiratory maneuvers in children aged 3 to 5 years. Pediatr Pulmonol 1994; 18: 144-9.

4. Aurora P, Stocks J, Oliver C, et al. Quality control for spirometry in pre-school children with and without lung disease. Am J Respir Crit Care Med 2004; 169: II52-9.

5. Arets HG, Brakel HJ, Van der Ent CK. Forced expiratory maneuvers in children: de they meet ATS and ERS criteria for spirometry? Eur Respir J 200 I; 1 8: 655-66.

6. Marostica PJ, Weist AD, Eigen $H$, et al. Spirometry in 3 to 6 year old children with cystic fibrosis. Am J Respir Crit Care Med 2002; |66: 67-7|.

7. Eigen $\mathrm{H}$, Bieler $\mathrm{H}$, Grant D, et al. Spirometric pulmonary function in healthy preschool children. Am J Respir Crit Care Med 200I; 163: 619-23.

8. Nystad W, Samuelsen SO, Nafstad P, Edvardsen E, Stensrud T, Jaakkola JJ. Feasibility of measuring lung function in preschool children. Thorax 2002; 57: 1021-7.

9. Vilozni D, Barker M, Jellouschek H, Heimann G, Blau H. An interactive computer animated system (SpiroGame) facilities spirometry in preschool children. Am J Respir Crit Care Med 200 I; 164: 2200-5.

I0. Jones MH, Davis SD, Grant D, Christoph K, Kisling J, Tepper RS. Forced expirator maneuvers in very young children. Assessment of flow limitation. Am J Respir Crit Care Med 1999; 159: 791-5.

11. Crenesse D, Berlioz M, Bourrier T, Albertini M. Spirometry in children aged 3 to 5 years: reability of forced espiratory maneuvers.
Pediatr Pulmonol 200I; 32: 56-6I.

12. Zapletal A, Chalupova J. Forced expiratory parameters in healthy preschool children (3-6 years of age). Pediatr Pulmonol 2003; 35: 200-7.

13. Nève $V$, Déme JL, Devos $P$, et al. Spirometry in 3-5 year-old children with asthma. Pediatr Pulmonol 2006; 41: 735-43.

14. Pérez-Yarza EG, Villa JR, Cobos N, et al. Forced spirometry in healthy preschool children. An Pediatr (Barc) 2009; 70: 3-II.

15. Linares M, Contreras I, Cox PP, Burgos P, Lara J, Meyer R. Evaluación del rendimiento de la espirometría en preescolares sanos con estandarización adaptada a este grupo etario. Rev Chil Enf Respir 2006; 22: 155-63.

16. Chavasse R, Johnson P, Francis J, Balfour-Lyn I, Rosenthal M, Bush A. To clip or not to clip? Noseclips for spirometry. Eur Respir J 2003; 21 : 876-8.

17. Gracchi V, Boel M, van der Laag J, van der Ent CK. Spirometry in young children: should computer-animation programs be used during testing? Eur Respir J 2003; 21 : 872-5.

18. Linares M, Meyer R, Cox PP, Contreras I. Utilidad del VEF $F_{0,5}$ versus $V E F$, en la interpretación de la espirometría realizada en preescolares asmáticos. Rev Chil Enf Respir 2007; 23: 167-72.

19. Quanjer PH, Stanojevic S, Cole TJ, et al. Multi-ethnic reference values for spirometry for the 3-95-yr age range: the global lung function 2012 equations. ERS Global Lung Function Initiative. Eur Respir J 2012; 40: 1324-43.

20. Debley J, Stanojevic S, Filbrun A, Subbarao P. Bronchodilator responsiveness in wheezy infants and toddlers is not associated with asthma risk factors. Pediatr Pulmonol 20 I 2; 47 (5): 42 I-8. 